\title{
Auto-Modification of Poly (ADP-Ribose) Polymerase and Membrane Lipid Composition as Diagnostic Tools to Measure the Physio-Pathological State of the Cell
}

Anna Rita Bianchi ${ }^{1}$, Simona Ruggiero ${ }^{2}$, Cesare Formisano ${ }^{2}$, Giuseppe Galloro ${ }^{2}$, Anna De Maio ${ }^{1}$, Carla Ferreri ${ }^{3}$ and Maria Rosaria Faraone Mennella ${ }^{1 *}$

${ }^{1}$ Departments of Biology, The University of Naples Federico II, Naples, Italy

${ }^{2}$ Departments of Clinical Medicine and Surgery, The University of Naples Federico II, Naples, Italy

${ }^{3}$ Consiglio Nazionale delle Ricerche-ISOF, Bologna, Italy

\begin{abstract}
Poly(ADPribosyl)ation, catalysed by poly(ADP-ribose)polymerases, affects many cellular events and has a recognized epigenetic role. Nuclear poly(ADP-ribose)polymerases 1 and 2 are hyper activated by DNA strandbreaks. They auto-modify with large polymers of ADP-ribose and recruit DNA repair proteins. The more the DNA strand-breaks, the more poly(ADP-ribose) polymerase modifies itself.

On the other hand, dietary lipids can be signaling molecules, lead to pro-( $\omega 6) /$ anti-( $\omega 3)$ inflammatory compounds and be included in biomembranes, good biomarkers of their unbalance.

Here, we report the results obtained from an epidemiological study to establish whether the combination of two different analyses, i.e. detecting auto-modified poly (ADP-ribose) polymerase levels and analyzing erythrocyte membrane fatty acid composition, might help to monitor the physio-pathological state of the cell, and to correlate with lifestyle, diet or diseases. The two analyses were carried blindly on 70 subjects undergoing endoscopy. They were first interviewed, to collect anamnesis and clinical data, if present. Lymphocytes and erythrocytes were prepared from venous blood to assay poly (ADP-ribose) polymerase auto modification and membrane fatty acid content, respectively. The results were statistically evaluated. The measure of poly (ADP-ribose) polymerase automodification confirmed that its levels correlate with DNA damage extent, within the same pathology, and allowed to monitor the clinical activity of the disease, depending on ongoing therapeutic/surgical treatment. Membrane fat profile was able to evidence unbalance of lipids linked to both diet/lifestyle and inflammatory states leading to diseases. Both analyses provide possible biomarkers for sensible, non-invasive and routine monitoring.
\end{abstract}

Keywords: Diet; Epigenomic; Niacin; Poly(ADP-Ribose) polymerases; Lipidomic

\section{Introduction}

Remodeling of chromatin is among epigenetic factors regulating gene expression [1]. Epigenetic changes have also been observed to occur in response to environmental exposure [2]. Unlike behavior or stress, diet is one of the better understood, environmental factors in epigenetic change [3].

There are increasing evidence that deleterious processes as membrane lipid peroxidation and DNA damage might be modulated by a correct quality of food intake and by post-translational modification reactions (namely NAD-dependent poly(ADP-ribosyl) ation), respectively.

The pyridine nucleotide Nicotinamide Adenine Dinucleotide $\left(\mathrm{NAD}^{+}\right)$mediates electron transfer in many catabolic processes [47], leading to ATP production. Thus, in addition to ATP, NADH is a potential high-energy carrier.

There are a number of enzymes using $\mathrm{NAD}^{+}$as substrate and synthesizing ADP-ribose (ADPR) and derivatives by cleavage of the bond between ADPR and nicotinamide. There are enzymes such as poly(ADP-ribose) polymerases (PARPs) that consume $\mathrm{NAD}^{+}[8-$ 10]. Among PARP family proteins, PARP-1 leads to depletion of $\mathrm{NAD}^{+}$pools in cells upon hyperactivation, and attenuates $\mathrm{NAD}^{+}$ dependent ATP production, resulting in cell death. PARP-1 is mainly involved in repairing damaged $\mathrm{DNA}$, a $\mathrm{NAD}^{+}$-consuming process as a consequence of PARP1 hyperactivation that takes place in both physiological and pathological conditions [11]. Activated and DNA bound PARP-1 synthesizes long and branched polymers of ADPribose (PAR) and concentrates up to $90 \%$ of cellular PAR on its automodification domain. DNA bound PARylated PARP-1 (PAR-PARP) promotes destabilization of DNA-histone interactions to allow DNA repair machinery to access to the damaged site [12]. Strong evidence has been provided for a specific relationship between DNA damage and acute depression of cellular NAD+ levels. Also, PARP-1 has been shown to affect metabolic homeostasis.

Given the role of PARP as biomarker of DNA damage, and its recognized importance as a signal of oxidative stress [10-12], it is conceivable that its activity levels/expression in the cell nucleus can help to define the physio-pathological state of the cell.

Lipids and phospholipids are very sensitive to stress conditions such as the reactive oxygen species (ROS) attack. The latter leads to change

*Corresponding author: Maria Rosaria Faraone Mennella, Professor of Biochemistry, Department of Biology, University of Naples "Federico II", Monte S. Angelo Campus, Building 7-Room 1F-35, Via Cintia - 80126 Naples, Italy, Tel: +39081679 ext. 136/135; E-mail: faraone@unina.it

Received March 04, 2014; Accepted May 05, 2014; Published May 09, 2014

Citation: Bianchi AR, Ruggiero S, Formisano C, Galloro G, De Maio A, et al (2014) Auto-Modification of Poly (ADP-Ribose) Polymerase and Membrane Lipid Composition as Diagnostic Tools to Measure the Physio-Pathological State of the Cell. J Microb Biochem Technol 6: 223-227. doi:10.4172/1948-5948.1000148

Copyright: (c) 2014 Bianchi AR, et al. This is an open-access article distributed under the terms of the Creative Commons Attribution License, which permits unrestricted use, distribution, and reproduction in any medium, provided the original author and source are credited 
Citation: Bianchi AR, Ruggiero S, Formisano C, Galloro G, De Maio A, et al. (2014) Auto-Modification of Poly (ADP-Ribose) Polymerase and Membrane Lipid Composition as Diagnostic Tools to Measure the Physio-Pathological State of the Cell. J Microb Biochem Technol 6: 223227. doi:10.4172/1948-5948.1000148

in contents of saturated (SFA), mono- (MUFA) and polyunsaturated (PUFA) fatty acids. Release of PUFA from membrane phospholipids triggers some physiological events [13-15]. Therefore the knowledge of the membrane status can be very important for the evaluation of the overall metabolic functioning [16]. The important role of changes to the membrane structure and corresponding physical-chemical properties are established [17]. Membranes are compositionally distinct in different tissues and are among the most exposed cellular components to oxidative stress. Lipid oxidation caused by ROS affects membrane proteins and reorganize membrane lipids, thus, leading to pathological conditions. That involves an increase of membrane fluidity and permeability, which arises from changes in lipid packing and molecular redistribution [18-20].

On the basis of these observations PARP automodification and membrane fatty acid composition are molecular aspects which can be taken as the mirror of the healthy/pathological state of the cell.

Our working hypothesis is that the combination of lymphocyte PARP automodification measure with erythrocyte membrane lipid profile, allows drawing either prognostic or diagnostic clinical picture to support therapeutic and/or nutraceutical approaches. Despite many evidence available in the literature suggest that increased oxidative stress and genomic instability can be monitored as early signals for prevention, these molecular aspects have not been examined in epidemiological and clinical settings yet.

Here, we present results from studies combining the two analyses to test the physio-pathology of cells by using venous blood as source of erythrocytes (for membrane fatty acid profile) and lymphocytes (for detection of automodified PARP levels). These data were compared with both antropometric measures (body mass index) and any clinical state of subjects undergoing the survey.

\section{Materials and Methods}

\section{Subjects}

The volunteers (70) object of this survey, ageing 30-75 years, were recruited at the Department of Clinical Medicine and Surgery for interview, blood collection and routine diagnostic and clinical analyses. They came from the same geographic area and used to follow a typical Mediterranean diet. They underwent endoscopies either to confirm or to get diagnosis of intestinal pathologies. They all signed an informed consent for anonymous treatment of their data, according to the privacy rules.

\section{Body mass index}

Body mass index (BMI) measured body fat based on height and weight of adult men and women to screen for weight categories that may lead to health problems, classified as under, normal, over-weight and obese. It was calculated as the ratio between weights in kilograms divided by the square of the height in meters $\left(\mathrm{kg} / \mathrm{m}^{2}\right)$. The three groups of subjects in this study were normal- weight (NW), over-weight (OW), and obese $(\mathrm{O})$.

\section{Blood collection and treatment}

Blood $(8 \mathrm{ml})$ was collected by venipuncture in the presence of EDTA and aliquoted. One aliquot $(4 \mathrm{ml})$ was used within few hours to prepare lymphocyte fraction. The second aliquot was used for erythrocyte membrane fatty acid composition.

\section{Lymphocyte preparation and lysis}

All operations were performed at $4^{\circ} \mathrm{C} .4$ blood aliquots $(1 \mathrm{ml})$ of each sample were separately used to prepare lymphocytes, after counting the cells per $\mathrm{ml}$ of blood by hematic cell counter. In this way taking into account a comparable loss of lymphocytes during preparation under the same conditions for all samples, the results were referred to the initial number of cells $/ \mathrm{ml}$.

Lymphocytes were prepared according to GE Healthcare protocol, provided with Ficoll. Briefly each blood aliquot $(1 \mathrm{ml})$ was layered on a Ficoll-Hypaque (GE Healthcare) cushion (1:0.7, v/v) and centrifuged at 2,500 rpm for 10 minutes. Because of their lower density, the lymphocytes were found at the interface between the plasma and the Ficoll-Paque PLUS with other slowly sedimenting particles (platelets and monocytes). The lymphocytes were recovered from the interface and subjected to short washing steps to remove any platelets, FicollPaque PLUS and plasma. Crude lymphocyte fraction was washed twice with $0.9 \% \mathrm{NaCl}$, followed by 10 min centrifugation at $1,500 \mathrm{rpm}$. Pelleted pure lymphocytes were suspended in $0.9 \% \mathrm{NaCl}(100 \mu \mathrm{l} / \mathrm{blood}$ $\mathrm{ml}$ ). Few microliters of the suspension were used for counting pure lymphocytes. In general a $20 \%$ loss was measured.

Pure lymphocytes were often used as freshly prepared fraction or stored at $-80^{\circ} \mathrm{C}$ until used.

Cells from $1 \mathrm{ml}$ blood were lysed by suspension in lysis buffer (300 $\mu \mathrm{l} ; 10$ mM Tris- $\mathrm{HCl}$ pH 7.5, 1\% Nonidet P40, 2 mM Spermidine$\mathrm{HCl}, 10 \mathrm{mM} \mathrm{Na}{ }_{2}$ EDTA, protease inhibitor cocktail $2 \mu \mathrm{g} / \mathrm{ml}$ (Sigma), $1 \mathrm{mM}$ Phenyl Methyl Sulphonyl Fluoride, PMSF) and incubation for 30 minutes at $4^{\circ} \mathrm{C}$. The whole lysate was further analyzed. Protein content was determined by Bradford's reagent (BioRad) according to the provided instructions.

\section{SDS-PAGE and immunoblotting}

Electrophoresis and protein blotting were according to FaraoneMennella et al. [21]. Polyclonal anti-PARP1 catalytic site antibodies (H250, Santa Cruz) were used to evidence PARPs. The highly conserved catalytic domain allowed recognizing any PARP with these antibodies. Automodified PARP (PAR-PARP) was quantified by densitometric analyses of immunobands with a Chemidoc (Bio Rad) and expressed as optical density (OD, i.e. intensity of a band) $/ \mathrm{mm}^{2}$.

Alkali incubation of PAR-PARP was carried on in $10 \mathrm{mM}$ Tris- $\mathrm{HCl}$ $\mathrm{pH}$ 9.5, for three hours at room temperature.

\section{Membrane fatty acid determination}

An aliquot $(4 \mathrm{ml})$ of each blood sample was used for the fatty acid analysis of erythrocyte membrane performed by the procedure described in [22].

This procedure is effected by an automatism set up at Lipinutragen [23], a spin-off company of the Italian National Council of Research (CNR) in Bologna (Italy), connected with the calculation of the membrane unbalance index, which is not reported in this paper [24].

\section{Statistical analysis}

The data were statistically analyzed both with parametric and nonparametric ( $\chi^{2}$ tests)

\section{Results and Discussion}

\section{Body mass index}

The antropometric data of all subjects allowed calculating their 
Citation: Bianchi AR, Ruggiero S, Formisano C, Galloro G, De Maio A, et al. (2014) Auto-Modification of Poly (ADP-Ribose) Polymerase and Membrane Lipid Composition as Diagnostic Tools to Measure the Physio-Pathological State of the Cell. J Microb Biochem Technol 6: 223227. doi:10.4172/1948-5948.1000148

body mass indexes (BMI). On this basis three groups were identified: normal weight $(\mathrm{NW} ; 19<\mathrm{BMI}<25)$; overweight $(\mathrm{OW}$; $\mathrm{BMI}<30)$; obese $(\mathrm{O} ; \mathrm{BMI}>30)$.

\section{PARP automodification analysis}

Immunoblotting with polyclonal anti-PARP catalytic site antibodies allowed visualizing the enzyme. Figure 1 shows the patterns of unmodified PARP from three analyzed samples (Figure 1A, a', lanes 1-3). In the absence of automodification the immunoband corresponded to native PARP. In Figure 1A (b', lanes 1-3) three examples of automodified PARP (PAR-PARP) from other samples are shown. PAR- PARP gave signals above $113 \mathrm{kDa}$. The amount of automodification was measured by densitometry of the bands.

A worth noting result was that the PARP enzyme mainly involved in auto modification in lymphocytes was PARP2 $(62 \mathrm{kDa}$; Figure $1 \mathrm{~A}$, a'). This finding was confirmed by an experiment where PAR-PARP was excised from the gel and the slice incubated under alkali conditions. The bond between protein and poly-ADPribose is highly sensitive to alkali treatment. The polymer-free PARP was eluted, electrophoresed and re-analyzed by the same antibodies (Figure 1B); it gave a band shifted from high molecular weight (above $113 \mathrm{kDa}$ ), to $62 \mathrm{kDa}$, the molecular mass of PARP2 (Figure 1B, 1'-3').

The lymphocyte PARP was analyzed for all subjects and the results were grouped according to automodification levels: OD $\leq 20000 \mathrm{int} /$

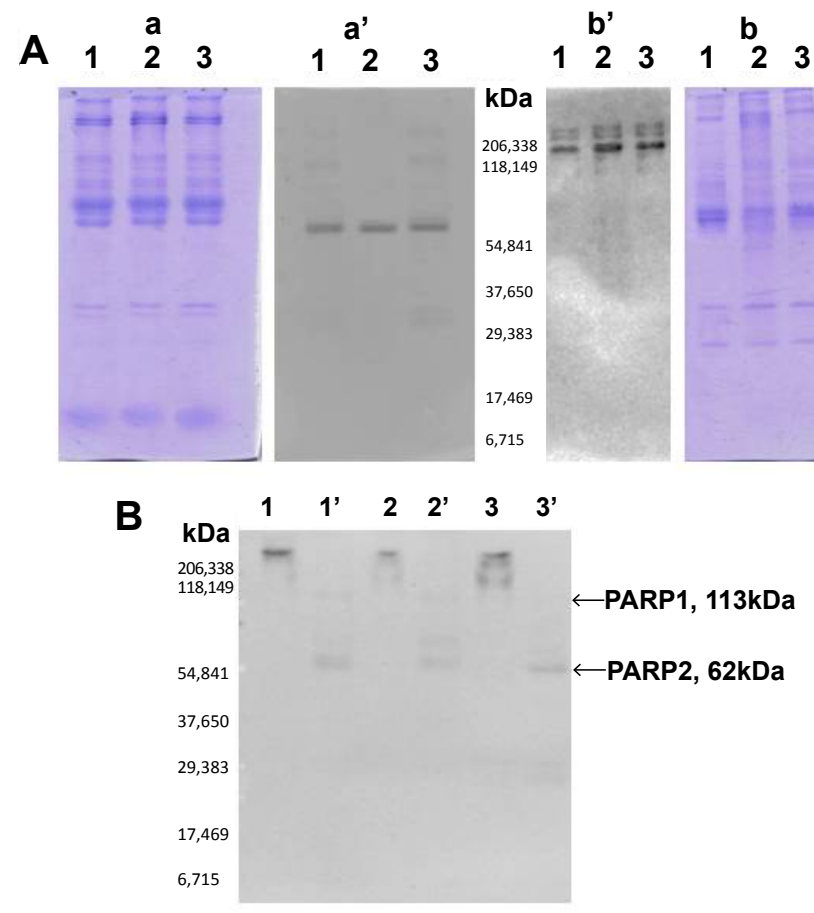

Figure 1: SDS PAGE and anti-PARP catalytic site immunoblotting of lymphocyte lysates.

Panel A.(a, b), Coomassie $(0.5 \%)$ stained gels of lymphocyte lysates; a', b', anti-PARP immunoblottings of the samples in $a, b$, respectively. In $a^{\prime}(1-3)$, the band of PARP was at $62 \mathrm{kDa}$, the molecular mass of PARP2 (native) for all three analysed samples. In b' (1-3) the immunobands was above $113 \mathrm{kDa}$, indicating a large automodification of PARP. The shift of molecular mass accounted for more than hundred ADPribose molecules modifying the enzyme Panel B. The immunopatterns in A, b', before $(1,2,3)$ and after (1', 2', 3') alkal incubation of PAR-PARP. In 1'- 3' poly-ADPribose-free PARP localizes at the molecular mass of native enzyme.

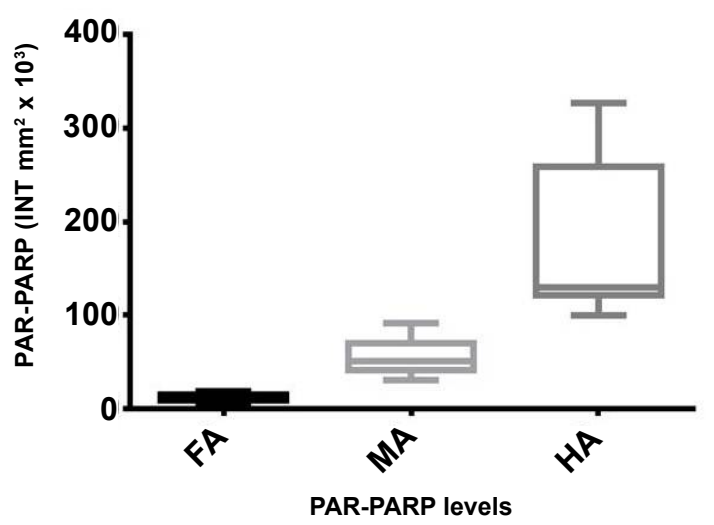

Figure 2: PARP automodification in analysed lysates.

FA, Physiological PAR-PARP $(n=24)$; MA, Moderate PAR-PARP $(n=36)$; HA High PAR-PARP $(n=10)$. Statistical significance of the difference between the three groups was calculated by analysis of variance, with $\mathrm{P}<0.01$.

\begin{tabular}{|c|c|c|c|}
\hline & NW & OW & 0 \\
\hline$\chi^{2}$ & 1.57 & 2.92 & 1.83 \\
\hline$n$ & 24 & 36 & 16 \\
\hline $\mathrm{P}$ & 1 & 1 & 0.999 \\
\hline
\end{tabular}

NW. Normal weight; OW. Over weight; O. Obese.

$n=$ number of cases

Table 1: $\mathrm{X}^{2}$ non parametric test of PAR-PARP compared to BMI of subjects.

$\mathrm{mm}^{2}$ corresponded to physiological PAR-PARP (FA); $20000 \leq \mathrm{OD} \leq$ $40000 \mathrm{int} / \mathrm{mm}^{2}$ indicated moderate levels of PAR-PARP (MA); hypermodification of PAR-PARP (HA) gave OD $>40000 \mathrm{int} / \mathrm{mm}^{2}$ (Figure 2). The difference between FA, MA and HA was statistically significant as calculated by both analysis of variance and $\chi^{2}$ test (Table 1 ). Increased PAR-PARP levels were measured in case of inflammation, viral infections and cancers with a relevant clinical activity (no therapeutic treatment, presence of metastases). An opposite FA was measured in healthy and remitting patients.

The comparison of PAR-PARP levels with BMI groups (NW, $\mathrm{OW}, \mathrm{O})$ gave, either within each group or between the three groups, $\mathrm{P}$ values highly above 0.05 , i.e. the difference was not statistically relevant (Figure 3 ). $\chi^{2}$ test confirmed these results (data not shown). Therefore, DNA damage occurrence is independent from antropometric data, and is easily detectable by PAR-PARP measures, even before performing other more complex diagnostic analyses. Similar results were obtained, comparing PAR-PARP levels and the age of volunteers (data not shown). However, by grouping the examined subjects in three ranges of age (years $20-39 ; 40-59 ;>60$ ) and considering anamnestic data, the results of Table 2 were obtained. The youngest people (years 20-39) showed mainly moderate PAR-PARP, at the highest percentage (50\%) for allergy, and $25 \%$ for either smoking or dislipidemy/hypertension. The latter increased to $60 \%$ and $80 \%$ for the other two ageing groups (40-59, >60, respectively). High PAR-PARP (HA) had noticeable incidence at any age for adenomas, autoimmunity and HCV/Papilloma virus infections. It occurred in $15 \%$ of aged (years $>60$ ) patients with cancer.

Physiological levels of PAR-PARP were measured either in the absence of diseases, or in cases of diseases under remission or therapeutic treatments (data not shown). 
Citation: Bianchi AR, Ruggiero S, Formisano C, Galloro G, De Maio A, et al. (2014) Auto-Modification of Poly (ADP-Ribose) Polymerase and Membrane Lipid Composition as Diagnostic Tools to Measure the Physio-Pathological State of the Cell. J Microb Biochem Technol 6: 223227. doi:10.4172/1948-5948.1000148

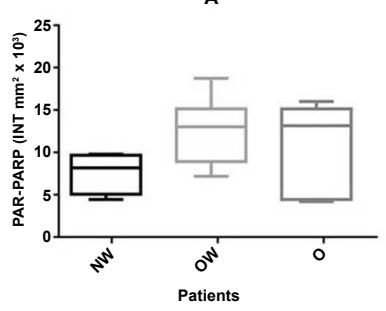

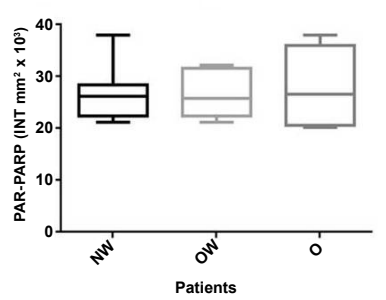

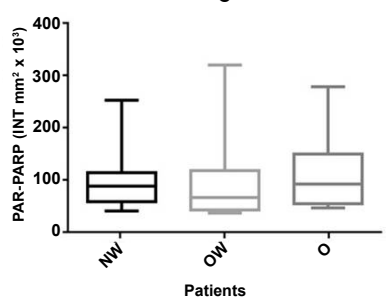

Figure 3: PAR-PARP levels compared with BMI groups.

$\mathrm{NW}=$ normal weight; $\mathrm{OW}=$ overweight; $\mathrm{O}=$ obese. The difference between groups was not significant $(\mathrm{P}>0.05)$.

\begin{tabular}{|c|c|c|c|c|c|c|}
\hline \multirow[b]{2}{*}{ Age (Years) } & \multicolumn{3}{|c|}{ MA } & \multicolumn{3}{|c|}{ HA } \\
\hline & $20-39(\%)$ & $40-59(\%)$ & $>60(\%)$ & $20-39(\%)$ & $40-59(\%)$ & $>60(\%)$ \\
\hline No Pathology & 25 (Smokers) & & & 10 (Obese) & & \\
\hline Allergy & 50 & 40 & 20 & 27 & & \\
\hline Hypertension/Dyslipidemias & 25 & 60 & 80 & & & \\
\hline Fibromatosis/Polyposis & & & & 27 & 50 & 50 \\
\hline Thyroid disease/Autoimmunity & & & & 27 & 30 & 15 \\
\hline Viral Infections & & & & 9 (HPV) & 20 (HCV) & $20(\mathrm{HCV})$ \\
\hline Cancer & & & & & & 15 \\
\hline
\end{tabular}

Percentage (\%) refers to the number of affected subjects per each group of PAR-PARP levels.

Table 2: Moderate (MA) and High (HA) PAR-PARP, and age-related pathologies/lifestyle of recruited subjects.
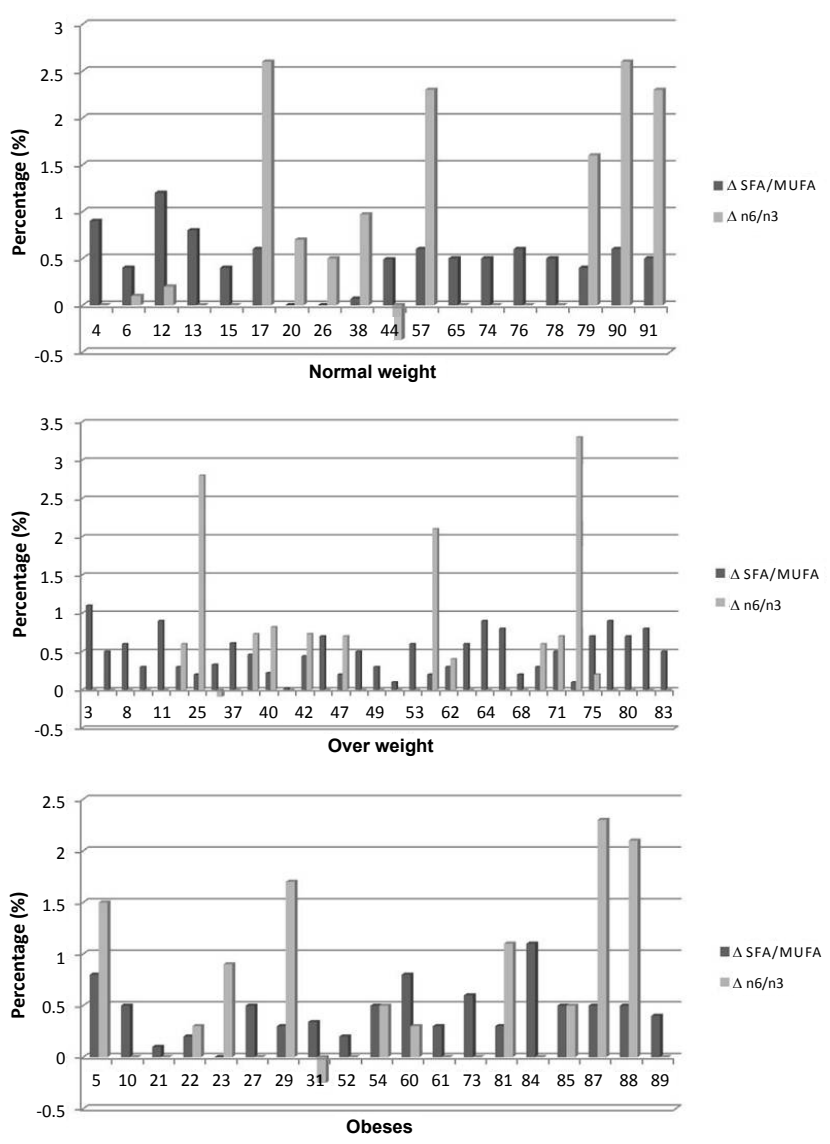

Figure 4: SFA/MUFA and omega-6/omega 3 PUFA unbalance in the recruited volunteers.

The difference of values measured for each patient versus normal values is reported. The three BMI groups (Normal Weight, NW; Over Weight, OW; Obeses, O) were considered.
It is worth noting that both moderate and high PAR-PARP levels were observed mostly in subjects with inflammatory conditions of lower or higher gravity (Table 2), but all relatable to different DNA damage extents $[5,8,11]$. As a routine analysis, PAR-PARP measure could be predictive of possible diseases involving DNA breakage.

Moreover, it is worth considering that increasing PARP automodification is strictly related to $\mathrm{NAD}^{+}$consumption. This reaction subtracts the pyridinic substrate from its coenzymatic function, and highly lowering its involvement in energy metabolism with deleterious consequences for ATP synthesis [4,9]. A relationship between PARP, DNA breakage, and niacin deficiency was reported previously [25].

Hence the importance of considering all pathways of NAD biosynthesis and recovery, but, essentially, checking the intake amount of the precursor niacin.

\section{Membrane fatty acid analysis}

By the described procedure the main fatty acids for the cell functions and metabolism were evaluated, including some important biomarkers such as the endogenous trans lipids, i.e., the lipids that are formed by the intervention of free radical species on cell membranes [22].

For the Italian population, the reference optimal values of erythrocyte membrane fatty acids have been described.

The parameters evaluated for the present study were the ratio of Saturated (SFA)/Monounsaturated (MUFA) Fatty Acids, and the ratio of omega-6/omega-3 Poly Unsaturated Fatty Acids (PUFA), given in Figure 4 . The figure shows the unbalance of fatty acids compared with normal values as measured for all recruited subjects, grouped for BMI, independently from disease occurrence. In any BMI group there was a strong unbalance of SFA/MUFA ratios compared to the control values (Table 3). The evidenced excess of SFA was an index of increased membrane rigidity, consequent altered permeability and functionality, with possible metabolic and cardiovascular risks [13,15,19]. These results at least allow correcting the quality of food, limiting the intake 
Citation: Bianchi AR, Ruggiero S, Formisano C, Galloro G, De Maio A, et al. (2014) Auto-Modification of Poly (ADP-Ribose) Polymerase and Membrane Lipid Composition as Diagnostic Tools to Measure the Physio-Pathological State of the Cell. J Microb Biochem Technol 6: 223227. doi:10.4172/1948-5948.1000148

\begin{tabular}{|c|c|c|c|}
\hline & NW & OW & 0 \\
\hline$\chi^{2}(\mathrm{~S} / \mathrm{M})$ & 1.91 & 2.3 & 0.84 \\
\hline$n$ & 12 & 17 & 7 \\
\hline $\mathrm{P}$ & 0.999 & 1 & 0.991 \\
\hline
\end{tabular}

NW. Normal Weight; OW. Overweight; O. Obese. $n=$ number of cases

Table 3: $\chi^{2}$ non parametric test of S/M ratio compared to BMI of subjects.

of nutritional sources of SFA, in order to re-establish the right content of these FA categories. Thereafter, it is possible to evaluate whether there might be alterations in any enzymatic step of FA metabolism requiring further attention.

A high unbalance was measured for omega-6/omega-3 PUFA. In some cases, there was a net increase of arachidonic acid, a precursor of many pro-inflammatory compounds, compared to the expected normal ranges.

Mostly a high reduction of omega-3 PUFA was observed. Due the anti-inflammatory value of these FA, their deficiency indicated a low anti-oxidant defense ability of cells, and a high risk of inflammation.

In conclusion, the results described in this paper for the first time compare DNA damage levels, monitored by PAR-PARP levels, and membrane lipid balance, with anamnestic data of subjects in order to study the relevance of these measures to evaluate the physio-patological state of the cell and if possible, to define dietary intervention in parallel with specific therapies. The healthy subjects of the 70 examined people had low PAR-PARP levels indicating a physiological DNA damage that occurs during normal metabolic events such as DNA replication, transcription, etc. However, they showed altered levels of membrane saturated fatty acids and $\omega 3$-PUFA which, on the basis of anamnestic data, might be attributable to incorrect diet and/or bad life habit (smoking). Similarly, in case of diagnosis of serious diseases, confirmed by high levels of PAR-PARP, people even in a normal BMI range, showed abnormal increase of SFAs, and a high deficiency of $\omega 3$-PUFA, i.e. of anti-inflammatory molecules. The knowledge of the actual status of a subject allows defining a dietary regimen to equilibrate the membrane lipids and to support specific therapies. It is worth noting that PARP automodification can be used widely as biomarker, for instance in the diagnostic of infiltrating inflammatory cells during stroke, myocardial and other forms of reperfusion injury, myocardial hypertrophy and heart failure, cardiomyopathies, circulatory shock, cardiovascular aging, atherosclerosis and vascular remodeling after injury, diabetic complications, and neurodegenerative disorders, since there is a wide literature about involvement of PARP in all these pathologies [25-27].

In combination the two analyses seem to contribute to draw at least diagnostic clinical pictures to support therapeutic and/or nutraceutical approaches.

\section{Acknowledgements}

The authors wish to thank the analytical support given by Dr. V. Sunda and Mr. S. Deplano in the analysis of erythrocyte membrane fatty acids at Lipinutragen srl (Bologna, Italy)

\section{References}

1. Jablonka E, Raz G (2009) Transgenerational epigenetic inheritance: prevalence, mechanisms, and implications for the study of heredity and evolution. Q Rev Biol 84: 131-176.

2. Reik W (2007) Stability and flexibility of epigenetic gene regulation in mammalian development. Nature 447: 425-432.

3. Waterland RA, Jirtle RL (2003) Transposable elements: targets for early nutritional effects on epigenetic gene regulation. Mol Cell Biol 23: 5293-5300.

4. Ying W (2006) NAD+ and NADH in cellular functions and cell death. Front Biosci 11: 3129-3148.

5. Berger $F$, Ramírez-Hernández MH, Ziegler $M$ (2004) The new life of a centenarian: signalling functions of NAD(P). Trends Biochem Sci 29: 111-118.

6. Khan JA, Forouhar F, Tao X, Tong L (2007) Nicotinamide adenine dinucleotide metabolism as an attractive target for drug discovery. Expert Opin Ther Targets 11: 695-705.

7. Stein LR, Imai S (2012) The dynamic regulation of NAD metabolism in mitochondria. Trends Endocrinol Metab 23: 420-428.

8. Mouchiroud L, Houtkooper RH, Auwerx J (2013) NADâ ${ }^{\circ}$ metabolism: therapeutic target for age-related metabolic disease. Crit Rev Biochem Mol Biol 48: $397-408$

9. Verdin E (2014) The many faces of sirtuins: Coupling of NAD metabolism sirtuins and lifespan. Nat Med 20: 25-27.

10. Bai P, Cantó C (2012) The role of PARP-1 and PARP-2 enzymes in metabolic regulation and disease. Cell Metab 16: 290-295

11. Luo X, Kraus WL (2012) On PAR with PARP: cellular stress signaling through poly(ADP-ribose) and PARP-1. Genes Dev 26: 417-432.

12. Mortusewicz O, Amé JC, Schreiber V, Leonhardt H (2007) Feedback-regulated poly(ADP-ribosyl)ation by PARP-1 is required for rapid response to DNA damage in living cells. Nucleic Acids Res 35: 7665-7675.

13. Chatgilialoglu C, Ferreri C, Melchiorre M, Sansone A, Torreggiani A (2014) Lipid geometrical isomerism: from chemistry to biology and diagnostics. Chem Rev 114: 255-284.

14. Cohen G, Riahi Y, Sunda V, Deplano S, Chatgilialoglu C, et al. (2013) Signaling properties of 4-hydroxyalkenals formed by lipid peroxidation in diabetes. Free Radic Biol Med 65: 978-987.

15. Ferreri C (2009) Trans Fatty Acids and Oxidative Transformations by Free Radicals: the Role in Health: Fatty Acids in Health Promotion and Disease Causation, Watson, R.R. ed, AOCS Press 379-405.

16. Ferreri C, Chatgilialoglu C (2012) Role of fatty acid-based functional lipidomics in the development of molecular diagnostic tools. Expert Rev Mol Diagn 12 767-780.

17. van Ginkel G, Sevanian A (1994) Lipid peroxidation-induced membrane structural alterations. Methods Enzymol 233: 273-288.

18. Simopoulos AP (2002) Omega-3 fatty acids in inflammation and autoimmune diseases. J Am Coll Nutr 21: 495-505.

19. Baritaki S, Apostolakis S, Kanellou P, Dimanche-Boitrel MT, Spandidos DA, et al. (2007) Reversal of tumor resistance to apoptotic stimuli by alteration of membrane fluidity: therapeutic implications. Adv Cancer Res 98: 149-190.

20. Calder PC (2009) Polyunsaturated fatty acids and inflammatory processes: New twists in an old tale. Biochimie 91: 791-795.

21. Faraone-Mennella MR, Marini M, Ferone A, Cacace O, Liguoro A, et al. (2010) Physical exercise activates the poly(ADP-ribosyl)ation system in rat testes. Biol Regul Homeost Agents 24: 325-333.

22. Ghezzo A, Visconti P, Abruzzo PM, Bolotta A, Ferreri C, et al. (2013) Oxidative Stress and Erythrocyte Membrane Alterations in Children with Autism: Correlation with Clinical Features. PLoS One 8: e66418.

23. Ferreri C (2008) Method for the evaluation of the functional status of cell membranes referred to the fatty acid components. EP2356468.

24. Zhang JZ, Henning SM, Swendseid ME (1993) Poly(ADP-ribose) polymerase activity and DNA strand breaks are affected in tissues of niacin-deficient rats. J Nutr 123: 1349-1355.

25. Pacher P, Szabo C (2008) Role of the peroxynitrite-poly(ADP-ribose) polymerase pathway in human disease. Am J Pathol 173: 2-13.

26. Pacher P, Szabó C (2007) Role of poly(ADP-ribose) polymerase 1 (PARP 1 ) in cardiovascular diseases: the therapeutic potential of PARP inhibitors. Cardiovasc Drug Rev 25: 235-260.

27. Geraets L, Moonen HJ, Brauers K, Wouters EF, Bast A, et al. (2007) Dietary flavones and flavonoles are inhibitors of poly(ADP-ribose)polymerase- 1 in pulmonary epithelial cells. J Nutr 137: 2190-2195. 